\title{
Meningoventriculitis Caused by Streptococcus pluranimalium in a Neonatal Calf of Premature Birth
}

\author{
Yukio M. SEIMIYA ${ }^{1)}$, Maki TAKAHASHI ${ }^{1)}$, Takashi KUDO ${ }^{1)}$ and Koji SASAKI ${ }^{1)}$ \\ ${ }^{1)}$ Iwate Prefecture Central Livestock Hygiene Service Center, 390-5 Sunagome, Takizawa-mura, Iwate 020-0173, Japan
}

(Received 20 October 2006/Accepted 19 February 2007)

\begin{abstract}
Pathological and bacteriological examinations were carried out on a neonatal calf that had developed nervous symptoms such as opisthotonus and blindness since it was born one month prior to full term. The principal lesions were characterized by fibrinopurulent inflammation of the meninges, choroid plexuses, and ventricular walls with limited extension to the subependymal parenchyma in the spinal cord. Purulent inflammation was also found in several visceral organs and tissues. Streptococcus pluranimalium was isolated from the brain and cerebrospinal fluid. These results suggest that the animal suffered from meningoventriculitis with septicemic $S$. pluranimalium infection.
\end{abstract}

KEY WORDS: meningoventriculitis, neonatal calf, Streptococcus pluranimalium.

Bacterial meningoventriculitis in neonatal calves is characterized by a unique surface-related inflammation of the meninges, choroid plexuses, and ventricular walls [1]. The disease is most often caused by Escherichia coli [1, 8, 10], and occasionally by Pasteurella [12], Klebsiella oxytoca [9], and Enterobacter cloacae [7]. Streptococcal infections, such as $S$. pneumoniae [5] and S. bovis [11], also produce meningoventriculitis concurrently accompanied by endophthalmitis. However, there seems to be no report describing the disease associated with $S$. pluranimalium in neonatal calves.

S. pluranimalium is a new streptococcal species designated in 1999 [2]. The organism has been isolated from lesions or normal organs of cattle and birds, such as milk of cows with subclinical mastitis and pox lesions of canaries as well as normal areas of the genital tract including the cervical and vaginal mucus in cows, the tonsils in calves, and the lungs and crop in canaries [2]. However, its pathogenic significance remains unclear [2]. This report describes the pathological and bacteriological findings in a neonatal calf suffering from meningoventriculitis with septicemic $S$. pluranimalium infection.

A neonatal calf was affected on a farm keeping 400 Japanese Black cows and 100 their calves. The calf was born at the fetal age of 252 days from the dam without any clinical symptoms in July 2005. The animal, weighed $19 \mathrm{~kg}$ at birth, had made a little attempt to suck colostrum and was fed small amounts of colostrum by the farmer. Nervous symptoms such as depression, inability in standing, opisthotonus, and blindness had been observed after birth. The animal did not respond to antibiotics treated on day-1 and 2 after birth, and was euthanatized due to unfavorable prognosis on day5.

Macroscopically, the cerebrospinal fluid was cloudy and increased in amount. Congestion, petechiae, and cloudy areas were seen in the meninges of the brain and spinal cord. There was a dilation of the ventricular system from the lat- eral ventricles to the spinal central canal, more severely in the lateral ventricles. A large amount of fibrinous exudate adhered to the ependymal surface of the dilated ventricular system, and was trapped among the folds of the choroid plexuses. The ventricular surface was discolored and roughened. In coronal sections of the cerebral hemispheres, laminar edematous lesions at the subependymal areas of the lateral ventricles extended approximately 1 to $4 \mathrm{~mm}$ into the deeper parenchyma (Fig. 1). In addition to the lesions in the central nervous system (CNS), there were miliary necrotic lesions in the liver and atrophy of the thymus.

Tissue samples collected from the CNS, eyes, and other representative organs and tissues were fixed in $10 \%$ neutral buffered formalin and embedded in paraffin wax. Paraffin

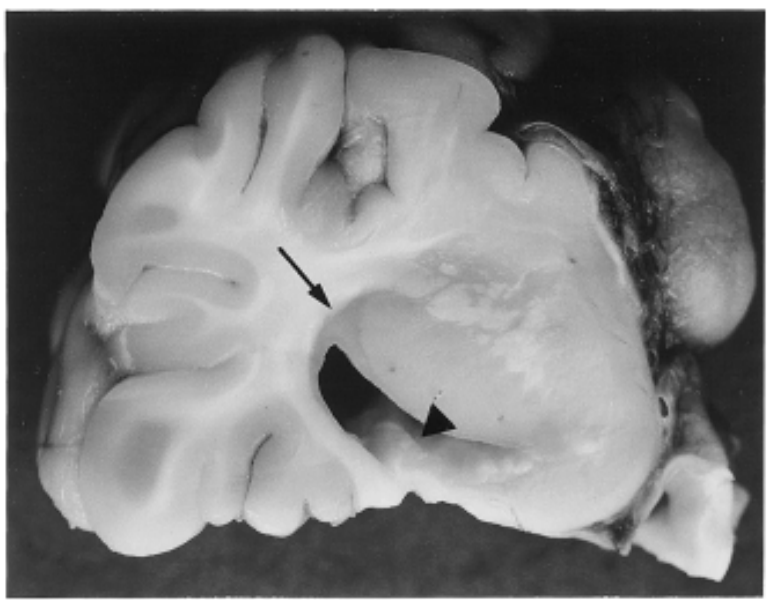

Fig. 1. Coronal section of the cerebral hemisphere at level of anterior basal ganglia, including the corpus striatum. There are fibrinous exudate (arrowhead) adhering to the surface of the dilated lateral ventricle and edema (arrow) at the subependymal area 


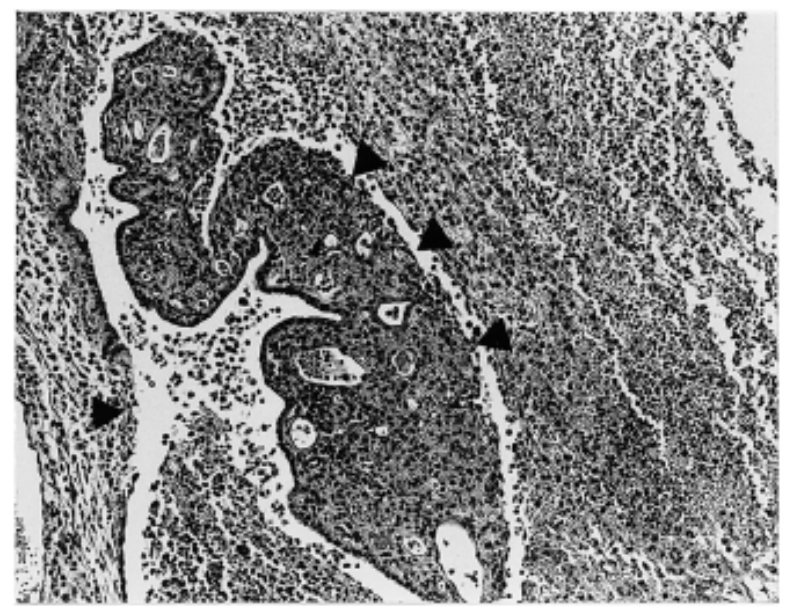

Fig. 2. Choroid plexus in the fourth ventricle. A large amount of fibrinopurulent exudate is trapped among the folds of the plexus. The epithelial cells are multifocally desquamated (arrowheads) with congestion and cellular infiltration in the stroma. HE stain. $\times 66$.

sections were stained with hematoxylin and eosin (HE). Selected sections of the CNS and representative visceral organs were stained with Gram (Brown and Brenn method), phosphotungstic acid hematoxylin, and azan.

The principal histological lesions were confined to the CNS. Fibrinopurulent inflammation involved the meninges, choroid plexuses, and ventricular walls with limited extension to the subependymal parenchyma in the spinal cord, and was more prominent in the choroid plexuses and ventricular walls. In the meninges, there were congestion, hemorrhages, fibrinous exudate, and infiltration of macrophages, neutrophils, and lymphocytes. The epithelial cells in all choroid plexuses were frequently desquamated along with congestion and infiltration of macrophages, neutrophils, and lymphocytes in the stroma (Fig. 2).

The ependymal cells were multifocally desquamated in most areas of the ventricular system (Fig. 3-A). At the subependymal zones, there were capillarization and mild proliferation of collagen fiber in and around the vascular adventitia together with infiltration of inflammatory cells. Rarefaction of the nerve tissue with swollen astrocytes extended from the subependymal zones to the middle or superficial layers of the white matter (Fig. 3-B), even to the deeper gray matter in the cerebral occipital lobes.

Infiltration of inflammatory cells into the rarefied nerve tissue was generally slight or mild. However, there were purulent lesions extending from the subependymal zone to the central intermediate substance in the first and second cervical segments of the spinal cord. The lesions consisted of rarefaction of the nerve tissue and neuronal degeneration together with focal and diffuse infiltration of neutrophils, macrophages, and lymphocytes. Capillarization and a few fibrinous thrombi could be seen also.

Numerous Gram-positive cocci with short chains were found in and around macrophages and neutrophils infiltrating into the purulent lesions of the CNS (Fig. 3-A, inset). Occasional colonies of bacteria were also present at stroma of the choroid plexus and subependymal areas. Other find-

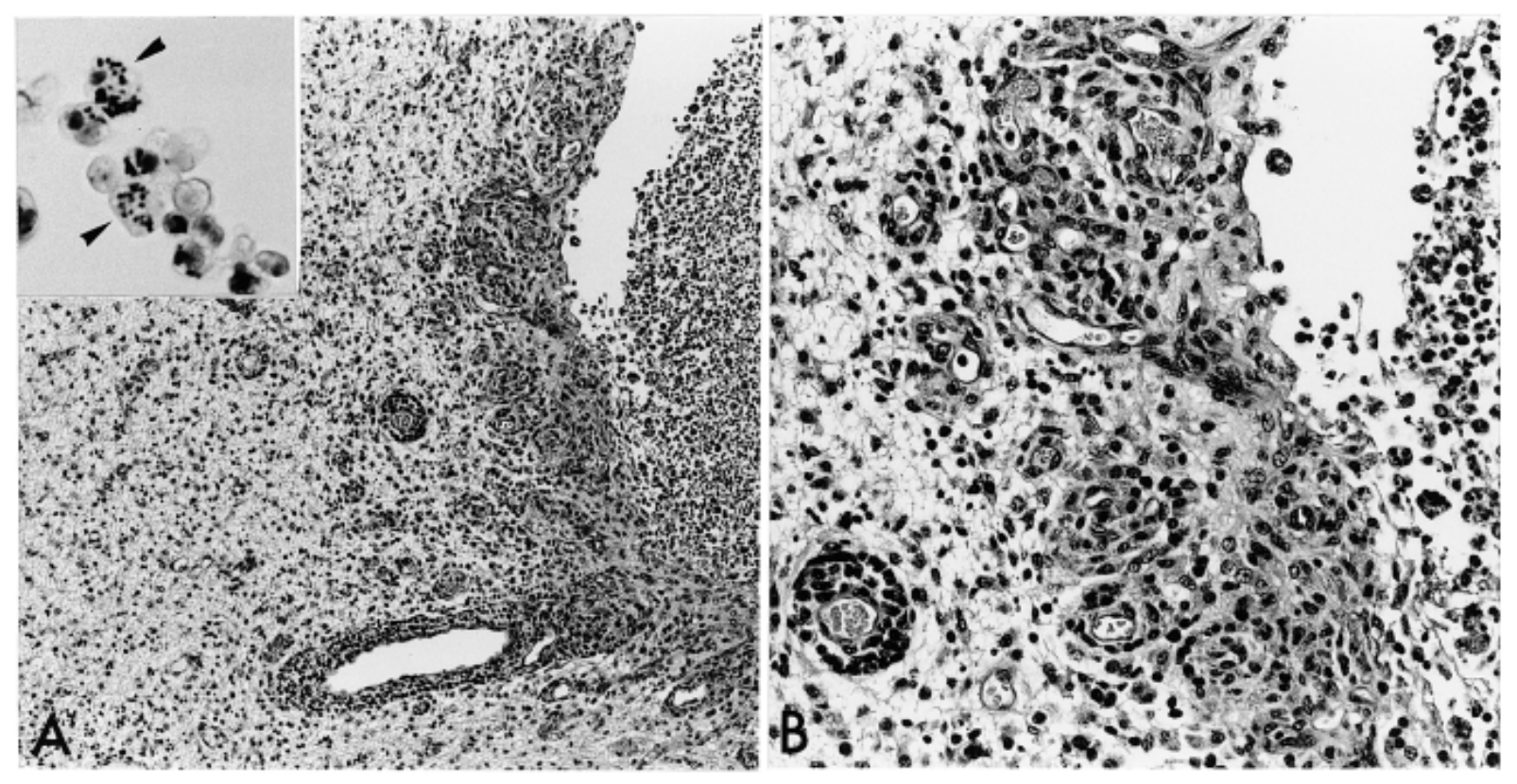

Fig. 3. Lateral ventricular wall lining cerebral parietal lobe. A) A large amount of fibrinopurulent exudate is seen on the ventricular surface where almost all ependymal cells are desquamated. HE stain. $\times 66$. Inset indicates Gram-positive cocci (arrowheads) in the infiltrated macrophages and neutrophils. Gram stain. $\times 660$. B) A higher magnification of A) showing capillarization and diffuse and perivascular cellular infiltration in the subependymal zone as well as rarefaction of the nerve tissue extending to the deeper cerebral white matter. HE stain. $\times 164$. 
ings were multifocal necrosis in the liver, fibrinopurulent peritonitis, and acute splenitis. There were no bacteria in purulent lesions in the organs and tissues other than the CNS. No lesions were found in the intraocular tissues.

Samples from the brain, cerebrospinal fluid, liver, spleen, kidneys, heart, and lungs were cultured aerobically on the tryptic soy agar supplemented with 5\% sheep blood, and anaerobically on Gifu anaerobic medium agar with 5\% egg yolk using a gas generating kit (Oxoid Ltd., England) at $37^{\circ} \mathrm{C}$ for $48 \mathrm{hr}$. The isolates were phenotypically and phylogenetically analyzed. The biochemical activities of the isolates were examined using a commercial system (API 20 Strep, bioMerieux, France). A fragment of the 16S rRNA genes (corresponding to positions 11-1544 of the E. coli) of the isolates was amplified by polymerase chain reaction with the primers forward F2 and reverse R13 [3], and sequenced directly using the BigDye terminators v1.1 cycle sequencing kit (Applied Biosystems, CAL, U.S.A.) and the ABI Prism 3,100 genetic analyzer (Applied Biosystems, CAL, U.S.A.). The sequences of the closest relatives of the isolates were retrieved from DNA databases by using BlastN program network service available at the National Center for Biotechnology Information, Bethesda, Md. (http://www.ncbi.nlm.nih.gov).

Both aerobic and anaerobic cultures yielded the colonies from the brain and cerebrospinal fluid, but not from other materials as mentioned below. The colonies on the blood agar were alpha-hemolytic and surrounded by pronounced greening zones with sharply demarcated edges. The isolates were Gram-positive cocci arranged in chains and reacted positively in the following tests: hippurate hydrolysis, $\beta$ glucuronidase and alkaline phosphatase activity, and acid production from trehalose. All were negative for VogesProskauer test and acid production from L-arabinose, glycogen, inulin, mannitol, ribose, sorbitol, and starch. The commercial system profiles were $2-5-6-0-4-1-0$, and indicated that the isolates were $S$. acidominimus.

In the $16 \mathrm{~S}$ rRNA sequence analysis, 774 nucleotides were determined with 2 gaps and eight undetermined nucleotides. Sequence searches of the GenBank database showed that both nucleotide sequences of the isolates and S. pluranimalium strain (accession number Y18026) were almost identical with a variation of one nucleotide (homology of 99.9\%), a replacement of adenine of position 1002 by guanine.

Both phenotypic and phylogenetic analyses are required for the accurate identification of the non-beta-hemolytic streptococci [4]. S. pluranimalium shares many phenotypic characteristics with $S$. acidominimus, but both strains are differentiated by the whole-cell protein or $16 \mathrm{~S}$ rRNA sequence analyses [2]. Alpha-hemolytic streptococci were isolated from the present premature calf. The phenotypic characteristics of the isolates were identical with those of $S$. pluranimalium strain [2]. By the characteristics, S. pluranimalium strain can be readily differentiated from phylogenetically related and phenotypically similar species, and also from $S$. acidominimus strain with respect to hippurate hydrolysis, alkaline phosphatase activity, and acid produc- tion from trehalose [2]. The isolates and S. pluranimalium strain showed homology as high as $99.9 \%$ in the $16 \mathrm{~S}$ rRNA nucleotide sequence. Based on the obtained results, the isolates were identified as $S$. pluranimalium.

The organism was not isolated from the materials other than the brain and cerebrospinal fluid. The result might be attributable to the treatments with antibiotics, since similar results were obtained in the neonatal calves with meningoventriculitis due to $E$. coli $[8,10]$.

The principal lesions in the present calf were characterized by fibrinopurulent meningitis, choroid plexitis, and ventriculitis with purulent inflammation of several visceral organs and tissues. These results suggest that the animal suffered from meningoventriculitis with septicemic S. pluranimalium infection, and that the organism might have a potential to induce septicemia or meningitis in neonatal calves. A possibility has been pointed out that several of streptococcal infections causing meningitis in neonatal calves are intrauterine $[5,11]$. It may be possible that the present case was also a continuation of the intrauterine infection, since nervous symptoms had been observed after birth.

An extension of inflammation from the ependyma in bacterial ventriculitis of neonatal calves is usually limited to the immediate subependymal zone where edema and cellular infiltration are found in the mild or slight degrees [1]. Inflammatory lesions in the present calf extended from the ependyma to the deeper parenchyma in the upper segments of the spinal cord. In infections of neonates, macrophages carry bacteria to the meninges, choroid plexuses, and ventricular walls $[1,13]$. And the bactericidal capacity of macrophages of fetal animals has been shown to be lower than that of neonatal animals [6]. Although the accurate factors associated with the development of severe subependymal lesions remain unknown, macrophages of the present premature calf might be much lower bactericidal capacity than those of normal neonatal calves, and might allow severe bacterial infection followed by extension of inflammation into the subependymal parenchyma. The treatments with antibiotics might also contribute to the protraction of the disease course resulting in the lesional progression.

Subependymal edema in the present calf was also severe and extended even to the deeper gray matter in the cerebral occipital lobes. Severe subependymal edema has been found as a rare case in a calf affected with severe ventriculitis accompanied by hydrocephalus due to $E$. coli [8]. It seems likely that the present subependymal edema resulted from invasion of the cerebrospinal fluid from the ventricular walls devoid of the ependymal cells, and that the increasing pressure of the cerebrospinal fluid facilitated the invasion. Blindness observed in the present calf may be ascribed to severe edema in the cerebral occipital lobes, since no intraocular lesions were present.

Meningitis and endophthalmitis are frequently concurrent in the streptococcal infections of neonatal calves $[5,11]$. Although the accurate reasons why the present calf was devoid of intraocular lesions remain unclear, it seems 
unlikely that the treatments with antibiotics might prevent the development of intraocular lesions, since the lesions can be induced in the early stage following the infection [5]. Further studies will be needed to accumulate knowledge of the pathogenicity of $S$. pluranimalium in animals, especially in neonatal calves.

\section{REFERENCES}

1. Cordy, D. R. 1984. Vet. Pathol. 21: 587-591.

2. Devriese, L. A., Vandamme, P., Collins, M. D., Alvarez, N., Pot, B., Hommez, J., Butaye, P. and Haesebrouck, F. 1999. Int. J. Syst. Bacteriol. 49: 1221-1226.

3. Dorsch, M. and Stackebrandt, E. 1992. J. Microbiol. Methods 16: $271-279$.

4. Facklam, R. 2002. Clin. Microbiol. Rev. 15: 613-630.

5. Jubb, K. V. F. and Huxtable, C. R. 1993. pp. 385-390. In: Pathology of Domestic Animals, vol. 1, 4th ed. (Jubb, K. V. F., Kennedy, P. C. and Palmer, N. eds.), Academic Press, New
York.

6. Karthigasu, K., Reade, P. C. and Jenkin, C. R. 1965. Immunology 9: 67-73.

7. Matsuda, M., Nakamura, K., Kondou, M., Mukai, J., Umeshita, T. and Tada, K. 1988. J. Jpn. Vet. Med. Assoc. 41: 433-435 (in Japanese with English summary).

8. Sato, S., Ohshima, K., Ichikawa, M. and Minamidate, K. 1988. J. Jpn. Vet. Med. Assoc. 41: 49-53 (in Japanese with English summary).

9. Seimiya, Y., Ohshima, K., Itoh, H., Murakami, R. and Haritani, M. 1993. J. Vet. Med. Sci. 55: 141-143.

10. Seimiya, Y., Ohshima, K., Itoh, H., Ogasawara, N., Okutomo, M. and Tanaka, S. 1992. J. Vet. Med. Sci. 54: 767-768.

11. Seimiya, Y., Ohshima, K., Itoh, H., Ogasawara, N., Okutomo, M. and Tanaka, S. 1992. J. Vet. Med. Sci. 54: 871-874.

12. Shand, A. and Markson, L. M. 1953. Br. Vet. J. 109: 491-495.

13. Williams, A. E. and Blakemore, W. F. 1990. Neuropathol. Appl. Neurobiol. 16: 377-392. 